referida associação; as principais dificuldades pelas quais passou a entidade e que colocaram em risco sua existência; as conquistas obtidas em seu percurso e que têm viabilizado sua sobrevivência. Para a consecução do trabalho foi realizada revisão bibliográfica e desenvolvido levantamento de informações junto à entidade objeto da investigação. Concluiu-se que esse tipo de associação tem sido fundamental para o fortalecimento do segmento citrícola e que a Associtrus vem provocando, junto aos citricultores, mudanças na cultura organizacional, inclusive, na postura deles com relação ao associativismo no meio citrícola.

\title{
Palavras-chave:
}

Associtrus, Citricultura, Associativismo.

\section{SisteMA de INFORMAÇÃO VIRTUAL PARA APOIO À GESTÃO DE MPES - MICRO E PEQUENAS EMPRESAS}

João Luiz Franco*

\section{Introdução}

As micro e pequenas empresas (MPEs) desempenham um papel importante na economia nacional e mundial. A principal contribuição é a geração de empregos, aspecto bastante relevante na economia brasileira em geral, especialmente no meio rural. Além disso, quando bem sucedidas, podem constituir o embrião de médias e grandes empresas (AZOLINI; COSTA, 2006).

Muitas das micro e das pequenas empresas atuam na informalidade. Não dispõem de informações organizadas para auxiliar no gerenciamento das atividades, muitas vezes por falta de conhecimento e de ferramentas que auxiliem nesse processo. Muitas empresas, também, carecem de recursos para investir em tecnologia de informação, tanto para compra de equipamentos como para implementação de sistemas de informação. Atualmente, a ausência de um sistema de gestão é um fator preocupante, pois compromete a competitividade da organização, muitas vezes decretando precocemente seu fim.

Existem sensíveis diferenças entre as características das micro e das pequenas em confronto com as grandes empresas. Enquanto a grande empresa tem características de uma organização burocrática (impessoalidade, formalidade e administração profissional), a pequena empresa apresenta características opostas: o proprietário administra o negócio e centraliza o poder em torno de si, atua em diversas áreas, impõe suas características pessoais à empresa e aos funcionários, utiliza a intuição como principal base para a tomada de decisão, preocupando-se mais com os problemas do dia-a-dia e deixando de refletir sobre o planejamento estratégico da empresa (MOTTA; PEREIRA, 2004; MENDES; ESCRIVÃO FILHO, 2000).

*Mestre em Ciência da Computação (ICMC-USP), Doutor em Engenharia Hidráulica (EESCUSP), membro do Grupo de Pesquisa Multidisciplinar em "Gestão das Organizações" do Departamento de Ciências da Administração e Tecnologia da Uniara, Bolsista Funadesp, Docente da Uniara, UFSCar e Unesp-IGCE, Campus de Rio Claro. 
A administração não profissional é evidenciada pela seleção do pessoal administrativo e de produção que não obedece, na maioria das vezes, a critérios técnicos. Muitas vezes são aproveitados parentes ou conhecidos sem o mínimo de conhecimento técnico necessário à função que irão exercer. Diante das particularidades mencionadas, é importante avaliar técnicas e ferramentas de gestão que possam auxiliar o administrador das micro e pequenas empresas a produzir melhores resultados gerenciais. Na verdade, as micro e pequenas empresas não necessitam de procedimentos complexos nem de técnicas sofisticadas, mas de um bom sistema de informação para apoio à gestão, que lhes permita compreender e atuar sobre o ambiente (FERRARI, 1987; MENDES; ESCRIVÃO FILHO, 2000).

A gestão empreendedora das micro e pequenas empresas, com base em uma estrutura administrativa mais leve que a das grandes empresas, permite a introdução de alterações e adaptações com maior rapidez do que ocorre em grandes empresas. As micro e as pequenas empresas estão localizadas mais próximas de seus clientes, o que possibilita identificar rapidamente mudanças de demanda, além de permitir que os serviços sejam prestados com maior agilidade no atendimento às solicitações dos clientes. Em geral, possuem competências bem específicas. Os sistemas de comunicação nas micro e nas pequenas empresas também são menos formais. Isto pode estar associado à sua estrutura organizacional, que é mais simples, necessitando de menor número de unidades ou de funções administrativas. Como aspectos negativos podem ser citados: maior desconhecimento das modernas técnicas de gestão, falta de recursos humanos especializados, dificuldade em contratar serviços especializados, dificuldade em conseguir capital (ALVIM, 1998; MENDES; ESCRIVÃO FILHO, 2000).

O primeiro aspecto a ser destacado, quando o assunto é aquisição de tecnologia, refere-se à escassez de recursos financeiros nesse segmento. Assim, tratando-se especificamente de sistemas integrados de gestão - os ERPs (Enterprise Resource Planning) - jamais se cogita de implantação de projetos que exigem grandes investimentos a serem concluídos no prazo de dois anos, por exemplo, o que é comum no mercado das grandes empresas.

\section{ERP - Sistemas Integrados de Gestão}

ERPs são sistemas de informações que abrangem a necessidade de informação de toda a empresa; são capazes de integrar toda suagestão, agilizando o processo de decisão (WOOD JR, 1999). Eles controlam e fornecem suporte a todos os processos operacionais, produtivos, administrativos e comerciais da empresa. Todas as transações da empresa devem estar registradas para que as consultas extraídas do sistema possam refletir o máximo possível sua realidade operacional. (LISBOA; TOFANELI; JOIOSO, 2003).
No início da década de 90, os ERPs passaram a ser largamente utilizados pelas empresas. Nessa época, eram extremamente caros, viáveis somente para empresas de grande porte. Ao adotar um ERP, o objetivo básico não é colocar o software em produção, mas melhorar os processos de negócios usando tecnologia da informação. Segundo Mendes e Escrivão Filho (2002) o ERP fornece informações geradas a partir do processo operacional, para otimizar o dia-a-dia da empresa, permitir um planejamento estratégico mais seguro e garantir a flexibilidade para evoluir (CENTOLA; ZABEU, 1999). Segundo Lima (2003), a documentação e a contabilização dos processos, por intermédio do ERP, geram regras de negócios bem definidas e permitem controle mais rígido sobre pontos vulneráveis da atividade. Mendes e Escrivão Filho (2002) afirmam que as micro e pequenas empresas têm recursos escassos e muitas vezes não podem arcar com o trabalho de uma empresa especializada que oriente a aquisição de um ERP.

Segundo Prates (2002) um sistema de informação voltado à pequena empresa deve respeitar alguns quesitos: custo, tempo e qualidade, e destaca que as micro e as pequenas empresas brasileiras são muito sensíveis a preço. Passini e Bertotto (2002) revelam que, nas pequenas empresas, que não possuem sistema de informação, a maior preocupação é com a questão financeira que implica em desenvolver um sistema computacional, ressaltando que a carência não é de computadores, mas de sistemas de informação que proporcionem facilidades de gerência aos pequenos empresários.

Uma empresa com um sistema totalmente informatizado, funcionando eficiente e eficazmente, proporcionará grandes vantagens, seja em relação ao tempo otimizado, à organização, à facilidade de obtenção de informações, à previsão e a muitos outros aspectos que contribuirão para o sucesso da pequena empresa (BERALDI; ESCRIVÃO FILHO, 2000). As empresas de pequeno porte estão procurando adquirir recursos de tecnologia para tratamento de informação. Entretanto, a aquisição desses recursos não é planejada e eles não são bem aproveitados devido, principalmente: à falta de conhecimento de suas potencialidade, às dificuldades para a aquisição de itens complementares e à falta de treinamento para os usuários. Além disso, a tecnologia de informação é um recurso de difícil acesso para as micro e as pequenas empresas: seu custo de aquisição é considerado alto e existe grande dificuldade de adequação e utilização desses recursos. Outra informação importante é que as empresas de menor porte investem muito mais em hardware do que em software.

\section{Informatização nas micro e pequenas empresas}

O Sebrae (BEDÊ, 2003) apresenta um estudo realizado em micro e pequenas empresas (MPEs) do Estado de São Paulo para identificar o grau de informatização dessas empresas, o acesso delas à internet e as perspectivas de 
investimento de curto prazo, nessa área. De acordo com esse levantamento, $47 \%$ das empresas se encontram informatizadas, metade das quais possuindo apenas um microcomputador (com predominância de Pentium 1/2/3 e sistema operacional Windows). Quanto aos periféricos, $72 \%$ das MPEs informatizadas contam com impressoras a jato de tinta; 66\% com leitora de CD-ROM; 50\% com kit multimídia; 46\% com impressora matricial; 30\% com scanner; $29 \%$ com no-break; $22 \%$ com impressora a laser; $18 \%$ com leitor de código de barra e gravador de CD-ROM; $8 \%$ com web cam; e 3\% com projetor multimídia. O estudo ressalta, também, que a informatização é maior nas MPEs mais antigas, do setor industrial e nas de maior porte.

Quanto à utilização, $81 \%$ das MPEs informatizadas usam seus microcomputadores para montar banco de dados de clientes (cadastro), 72\% para elaborar documentos, 66\% para acessar serviços na internet, 56\% para controlar estoques, 51\% para controlar folha de salários/pagamentos, 50\% para enviar mala direta (fax ou e-mail), 48\% para realizar a automação de processos e $36 \%$ para emitir notas fiscais.

Os softwares mais empregados são os editores de texto, presentes em $81 \%$ das MPEs informatizadas, as planilhas eletrônicas (70\%), softwares feitos sob encomenda (43\%), pequenos sistemas de banco de dados pessoais $(39 \%)$, softwares para apresentações $(36 \%)$.

Independentemente de possuírem microcomputadores nas empresas, $54 \%$ dos empresários afirmaram ter acesso à Internet. Essa proporção é superior à das MPEs informatizadas $(47 \%)$ e pode ser explicada pelo fato de que o acesso pode ocorrer fora da empresa, em lugares como: domicílio do empresário, domicílio de parentes/amigos, cyber cafés, lojas de acesso à internet e de outras maneiras. Esses dados mostram que a falta de microcomputador não impede o acesso à internet $\mathrm{e}$ os benefícios que ela pode proporcionar.

Entre as MPEs que não possuem microcomputador, as razões apontadas para não utilizar esse tipo de equipamento são: não vêem necessidade nem benefício (64\%), alegam requerer elevado investimento (44\%), não sabem mexer em computador (10\%), não possuem empregados qualificados $(6 \%) \mathrm{e}$ outras razões $(2 \%)$.

Com relação a investimentos nos próximos 6 meses, 19\% dos entrevistados pretendiam realizar investimentos em informática, enquanto $49 \%$ não tinham a intenção de fazer esse tipo de investimento. Entre os que pretendiam realizar algum investimento em informática, 73\% mencionaram microcomputadores (novos e/ou na atualização dos já existentes) e 26\% softwares.

Segundo Carmo e Pontes (1999), programas de apoio às Pequenas e Médias Empresas (PMEs) são considerados como estratégicos para o desenvolvimento econômico não apenas nos países em desenvolvimento, mas também nos países centrais. A capacidade de as PMEs atuarem de forma crítica e competitiva junto à cadeia de mercado está relacionada à formulação de estratégias de negócios nas quais são levadas em consideração não apenas as ações e reações dos concorrentes diretos, mas também a existência de fornecedores, clientes e produtos alternativos que satisfaçam as necessidades dos mercados onde estas empresas atuam.

Assim, considerando-se o contexto apresentado com relação aos problemas enfrentados pelos empresários de empresas de menor porte, tornase importante a elaboração de um sistema de informações voltado para a realidade dessas empresas, tendo por objetivo proporcionar uma ferramenta de apoio ao gerenciamento estratégico das mesmas, sobretudo das micro empresas, contribuindo para seu desenvolvimento, melhoria da qualidade e produtividade. Este procedimento viabiliza aos empresários o acompanhamento mais rápido e conveniente das informações relevantes para o gerenciamento (CARMO; PONTES ,1999).

No caso das micro empresas, as incubadoras de empresas constituem um locus privilegiado para o aprofundamento do estudo pretendido, testando a operacionalidade do sistema proposto.

\section{Incubadora de empresas de Araraquara-SP}

A incubadora de empresas selecionada foi a de Araraquara-SP, em razão de facilidade de acesso e da possibilidade de avaliação e acompanhamento dos resultados observados.

O município de Araraquara-SP é sede da Região de Governo de mesmo nome e, juntamente com a Região de Governo de São Carlos, compõe a Região Administrativa Central do Estado de São Paulo. Com população estimada para 2006 de 195.844 habitantes, ocupa área de $1011 \mathrm{~km} 2$ e apresenta taxa de urbanização para 2006 de 95,93. (FUNDAÇÃO SEADE).

Dentre as atividades industriais destacam-se os setores mecânico, metalúrgico, têxtil, de produtos alimentares e de vestuário.

Comércio e Serviços são responsáveis pelo emprego de $60 \%$ da mãode-obra formalmente empregada no município.

A agroindústria, representada pelo binômio cana e laranja, ocupa significativo papel na economia do municípioe da região. O setor sucroalcooleiro conta com três usinas de açúcar e álcool no município de Araraquara e quinze num raio de 80 quilômetros. Tem sede em Araraquara a maior empresa do país na produção de Suco de Laranja Concentrado Congelado (SLCC) que é, também, uma das maiores exportadoras no mundo. Juntamente com outras quatro grandes produtoras, também instaladas na região, responde por $96 \%$ da produção brasileira de suco de laranja concentrado congelado (SLCC). 
São as seguintes as informações sobre o número de empresas no município de Araraquara. Os dados têm o ano base 2005 (últimos dados disponíveis) e incluem apenas empresas legalizadas, por setor de atividade e tamanho das empresas, considerando o número de empregados.

Quadro 1. Empresas do Município de Araraquara-SP, distribuídas por Setor de Atividade e tamanho da empresa (micro, pequena, média e grande), por número de empregados registrados (2005).

\begin{tabular}{|c|c|c|c|c|c|c|c|c|c|c|}
\hline & \multicolumn{3}{|c|}{$\begin{array}{c}\text { Miero } \\
\text { Empresa }\end{array}$} & \multicolumn{2}{|c|}{$\begin{array}{l}\text { Pequena } \\
\text { Empresa }\end{array}$} & \multicolumn{2}{|c|}{$\begin{array}{c}\text { Média } \\
\text { Empresa }\end{array}$} & \multicolumn{2}{|c|}{$\begin{array}{c}\text { Grande } \\
\text { Empresa }\end{array}$} & \multirow{2}{*}{ Total } \\
\hline & até 4 & $\begin{array}{l}\text { de } 5 \text { a } \\
9\end{array}$ & $\begin{array}{c}\text { de } 10 \mathrm{a} \\
19\end{array}$ & $\begin{array}{c}\text { de } 20 \mathrm{a} \\
49\end{array}$ & $\begin{array}{c}\mathrm{de} 50 \mathrm{a} \\
99\end{array}$ & $\begin{array}{l}\text { de } 100 \\
\text { a } 249\end{array}$ & $\begin{array}{l}\text { de } 250 \\
\text { a } 499\end{array}$ & $\begin{array}{c}\text { de } 500 \\
\text { a } 999\end{array}$ & $\begin{array}{c}1000 \text { ou } \\
\text { mais }\end{array}$ & \\
\hline I. Indústria & 483 & 96 & 58 & 35 & 13 & 4 & 7 & 1 & 1 & 698 \\
\hline 2. Construção civil & 287 & 28 & 18 & 8 & 2 & 3 & 0 & 0 & 0 & 346 \\
\hline 3. Comercio & 4.881 & 355 & 175 & 68 & 18 & 6 & 2 & 0 & 0 & 5.505 \\
\hline 4. Serviços & 3.439 & 251 & 153 & 82 & 25 & 14 & 8 & 3 & 2 & 3.977 \\
\hline $\begin{array}{l}\text { 5. Agropecuária, extr. } \\
\text { vegetal, caça e pesca }\end{array}$ & 445 & 23 & 12 & 8 & 3 & 2 & 3 & 1 & 2 & 499 \\
\hline Total & 9.535 & 753 & 416 & 201 & 61 & 29 & 20 & 5 & 5 & \\
\hline Total Geral & & 10.704 & & 2 & & 4 & & & 10 & 11.025 \\
\hline
\end{tabular}

Fonte: Quadro elaborado a partir de Relatório Anual de Informação Social (RAIS) - Ministério do Trabalho e Emprego (MTE).

Quadro 2. Distribuição dos empregados com vínculos ativos por setor de atividade, no Município de Araraquara-SP (2005).

\begin{tabular}{|l|r|r|r|r|r|r|}
\hline & Indústria & Construção Civil & Comércio & Serviços & Agropecuária & Total \\
\hline Nenhum vínculo ativo & 327 & 189 & 3.598 & 2.330 & 258 & 6.702 \\
\hline Até 4 vinculos ativos & 156 & 98 & 1.283 & 1.109 & 187 & 2.833 \\
\hline De 5 a 9 vinculos ativos & 96 & 28 & 355 & 251 & 23 & 753 \\
\hline De 10 a 19 vinculos ativos & 58 & 18 & 175 & 153 & 12 & 416 \\
\hline De 20 a 49 vinculos ativos & 35 & 8 & 68 & 82 & 8 & 201 \\
\hline De 50 a 99 vinculos ativos & 13 & 2 & 18 & 25 & 3 & 61 \\
\hline De 100 a 249 vinculos ativos & 4 & 3 & 6 & 14 & 2 & 29 \\
\hline De 250 a 499 vinculos ativos & 7 & 0 & 2 & 8 & 3 & 20 \\
\hline De 500 a 999 vinculos ativos & 1 & 0 & 0 & 3 & 1 & 5 \\
\hline 1000 ou mais vinculos ativos & 1 & 0 & 0 & 2 & 2 & 5 \\
\hline Total & $\mathbf{6 9 8}$ & $\mathbf{3 4 6}$ & $\mathbf{5 . 5 0 5}$ & $\mathbf{3 . 9 7 7}$ & $\mathbf{4 9 9}$ & $\mathbf{1 1 . 0 2 5}$ \\
\hline
\end{tabular}

Fonte: Quadro elaborado a partir de Relatório Anual de Informação Social (RAIS), Ministério do Trabalho e Emprego (MTE).
A Incubadora de Empresas de Araraquara é um projeto que tem por objetivo auxiliar novos empreendimentos, oferecendo acessoria jurídica, consultorias de custos e finanças, marketing e treinamentos periódicos. O tempo de permanência no projeto é de 3 anos e atualmente conta com 14 empresas instaladas em suas dependências, segundo o quadro a seguir:

Quadro 3. Empresas instaladas na Incubadora de Empresas de Araraquara em 2007.

\begin{tabular}{|c|c|c|}
\hline Nome & Segmento & Postos de Trabalho \\
\hline Brás Weld Ind. e Com de Soldas Ltda - EPP & Metal Mecânico & 4 \\
\hline Brasil Satélite Tecnologia em Equipamentos Ltda & Tecnologia & 5 \\
\hline Carrex Ind. e Com Ltda - ME & Metal Mecânico & 3 \\
\hline DPS - Ind. Com de Equipamentos Eletroeletrônicos Ltda & Tecnologia & 4 \\
\hline Daniel de Lima Faria Lida - ME & Destilaçĩo de Pinga & 2 \\
\hline MultiMeias Ind. e Com Ltda - ME & têxtil & 10 \\
\hline MBF Comercial Ltda-ME & Metal Mecânico & 4 \\
\hline Natural Rural Ind. e Com Lida - ME & Agronegócios & 9 \\
\hline Óltimo Alimentos Ltda - ME & Alimenticio & 3 \\
\hline Naturom Ind. Com de Organismos Microbiológicos Ltda & Agronegócios & 9 \\
\hline MGPAR Ind. e Com Ltda - ME & Metal Mecânico & 4 \\
\hline Pró Exata Automação Industrial Ltda & Metal Mecânico & 3 \\
\hline Primus Desenvolvimento WEB Ltda & Tecnologia (SI) & 4 \\
\hline IBX Ind. e Com Ltda & Metal Mecânico & 3 \\
\hline
\end{tabular}

Fonte: Incubadora de empresas de Araraquara-SP (2007).

O projeto auxilia também algumas empresas associadas, que no momento são: Luma Cardans, Teniza Revestimentos, MacLub e Alfa, além de duas empresas ex-incubadas: Aramold e Casual.

Desenvolvimento do sistema de gestão para micro e pequenas empresas

Uma das grandes revoluções na Internet foi caracterizada pela geração e obtenção de informações dinâmicas. Uma das mais importantes tecnologias para manipular informações dinâmicas são as Servlets, que constituem a base do desenvolvimento de aplicativos Web utilizando a linguagem de programação Java (BOTEGA; FRANCO; LEMES, 2006). Isto permite que muitos serviços sejam fornecidos via Internet, sem que seja necessário instalar um sistema na máquina do cliente. Sistemas implementados dentro dessa filosofia apresentam vantagens consideráveis:

a) a atualização dos sistemas é feita somente no servidor, não sendo necessário refazer a instalação em todos os computadores que utilizam o sistema;

b) não são necessários equipamentos de alta performance computacional, já que praticamente todo o processamento é realizada no servidor;

c) há economia nos dispositivos de armazenamento de dados, já que o Banco de Dados fica no servidor; 
d) não é necessário controle de backup, que será feito somente no servidor;

e) é possível acessar o sistema de qualquer lugar com acesso à Internet, de forma que um defeito no computador do cliente não impede que o sistema possa ser utilizado, bastando utilizar outro computador.

O desenvolvimento de um Sistema de Gestão para micro e pequenas empresas envolve testar as necessidades de gerenciamento de informação de empresas com esse perfil, com o objetivo identificar os problemas comuns enfrentados e também as necessidades específicas de algumas áreas. Assim sendo, a partir do diagnóstico das necessidades das micro empresas, esboçouse um sistema preliminar, menos detalhado, a ser apresentado às empresas. Após avaliação-teste por parte delas, propõe-se a construção de um Sistema de Informação, cuja característica principal seja atender às principais necessidades de gerenciamento da informação das micro e pequenas empresas, principalmente das primeiras, com um mínimo de investimento por parte dos empresários. Afinal, o investimento em um equipamento computacional para acesso à Internet é muito inferior ao necessário para utilização de um ERP. Além do mais, não serão necessários investimentos em manutenção do sistema e em segurança computacional, pois todos os procedimentos serão realizados no servidor.

Para isso o sistema será implementado utilizando a tecnologia Java Servlet, e será hospedado em um site, podendo ser acessado via Web a partir de qualquer computador conectado à Internet. $\mathrm{O}$ sistema será o mesmo para todas as empresas, e utilizará um Banco de Dados compartilhado para armazenar informações e permitir acesso remoto via WEB, mas deverá transmitir ao usuário a percepção de que ele possui um sistema independente gerenciando sua empresa. O sistema deverá permitir a inclusão, exclusão, alteração e consultas das informações, bem como a impressão de diversos relatórios. $\mathrm{O}$ acesso só será permitido a empresas cadastradas, após confirmação de senha.

A centralização das informações em um único Banco de Dados também facilitará a obtenção de informações sobre as diversas empresas usuárias do sistema, tanto individuais quanto agrupadas, possibilitando traçar um perfil mais detalhado das micro e pequenas empresas da região, facilitando a formulação de ações de política pública que estimulem o desenvolvimento regional.

Há também que se destacar a importância das diversas empresas estarem interligadas sob um único sistema, permitindo a formação de uma Comunidade Virtual de Empresas, que possam interagir e trocar experiências entre si, bem como facilitar o envio de informações para as diversas empresas da Comunidade Virtual.
Desta forma, a criação da Comunidade Virtual de Micro e Pequenas Empresas trará importantes benefícios para todos os envolvidos nesse projeto: as micro e pequenas empresas de Araraquara e Região, a Incubadora de Empresas de Araraquara, e toda a comunidade que, indiretamente, irão colher os frutos do fortalecimento empresarial na região.

\section{Considerações finais}

A importância e a necessidade do sistema proposto ficou comprovada pela análise da literatura referente às micro e pequenas empresas. Seu detalhamento, após avaliação-teste por parte das micro empresas, instaladas na incubadora de Araraquara apresenta viabilidade e procedência.

Além dos efeitos positivos da utilização do sistema, no curto e médio prazos, a criação da Comunidade Virtual de Micro Empresas poderá ultrapassar os limites da incubadora, estendendo seus benefícios num raio maior, favorecendo o melhor desempenho de empresas no âmbito do município e mesmo da região.

\section{Referências:}

ALVIM, P.C.R.C. O papel da informação no processo de capacitação tecnológica das micro e pequenas empresas. Ciência da Informação, Brasília, v.27, n.1, p.28-35, jan/abr., 1998

AZOLINI, V.; COSTA, V.M.H.M. A Utilização da Informação Contábil para a Gestão da Apicultura de Pequeno Porte. Revista Uniara, Araraquara, n. $17 / 18,2005 / 2006$.

BEDÊ, M.A. (Coord.). A informação nas MPEs paulistas. Relatório de pesquisa. São Paulo: SEBRAE, 2003.

BERALDI, L. C.; ESCRIVÃO FILHO, E. Impacto da tecnologia de informação na gestão de pequenas empresas. Ciência da Informação, Brasília, v.29, n.1, p. 46-50, 2000.

BOTEGA, B.M.; FRANCO, J.L.; LEMES, S.S. Desenvolvimento de Sistema para Avaliação On-Line com Tecnologia Java/Servlets. Revista Uniara, Araraquara, n.17/18, 2005/2006. 
BRASIL. Ministério do Trabalho e Emprego. Relatório Anual de Informação Social - RAIS. Brasília, DF, 2005.

CARMO, V.B.; PONTES, C.C.C. Sistemas de informações gerenciais para programa de qualidade total em pequenas empresas da região de Campinas. Ciência da Informação, Brasília, v.28, n.1, Jan. 1999.

CENTOLA, N.; ZABEU, S. B. Pequenas e médias empresas: tomem a rédea de seus negócios! PC WORLD, p. 34-54, mar. 1999.

FERRARI, M.M. Lamattina de diseño de estrategias y políticas para pequeñas y medianas empresas. Administración de Empresas, Bueno Aires, v. 18, n.213, p.647-60, 1987.

FUNDAÇÃO SEADE. Perfil municipal. Disponível em: www.seade.gov.br. Acesso em: 10 set. 2007

LIMA. A. D. A. et al. Implantação de pacote de gestão empresarial em médias empresas. Artigo publicado pela KMPress. 30/06/2003. Disponível em: http://www.kmpress.com.br. Acesso em: 02 mai. 2006

MENDES, J.V.; ESCRIVÃO FILHO, E. Sistemas integrados de gestão em médias empresas: estudo sobre a adoção em empresas da grande São Paulo. In: XI Encontro Nacional da ANGRAD, 2000, Salvador. Anais..., Salvador, 2000, p.104-113.

. Sistemas Integrados de Gestão ERP em pequenas empresas: um confronto entre referencial teórico e a prática empresarial. Gestão \& produção, São Carlos, v. 9, p. 277-296, 2002

MOTTA, F.C.P.; PEREIRA, L.C.B. Introdução à organização burocrática. São Paulo: THOMSON LEARNING, 2004.

LISBOA, F.O.S.S.; TOFANELI, C.; JOIOSO, A.L.B.J. Um sistema ERP Acadêmico na Intranet. Revista Uniara, n. 12, 2003.

PASSINI, J.M.; BERTOTTO, C.A. Metodologia de implantação de sistemas de Informação para micro e pequenas empresas no município de Santa Maria. 2002. Monografia (Bacharelado em Sistemas de Informação) - Centro Universitário Franciscano, 2002.
PRATES, G.A. Tecnologia da informação em pequenas empresas. analisando empresas do interior paulista. Administração on line prática, pesquisa, ensino, São Paulo, v.3, n.4, 2002.

WOOD JR., T. Modas e modismos gerenciais: o caso dos sistemas integrados de gestão. Série Relatórios de Pesquisa - Fundação Getúlio Vargas, São Paulo, n.16, 1999

\section{Resumo:}

Considerando as necessidades de MPEs (Micro e Pequenas Empresas) registradas pela literatura e por organismos de apoio, em especial a Incubadora de Empresas de Araraquara, a investigação que deu suporte ao presente trabalho pretende levantar necessidades de gerenciamento de informação em empresas selecionadas no município de Araraquara e desenvolver um sistema de informação para apoiar a gestão dessas empresas, aplicável nas demais de mesmo porte. O sistema deverá ser instalado em um servidor (que poderá ser administrado pela Uniara ou por instituição com quem estabeleça parceria) e poderá ser utilizado em qualquer computador acessando a Internet, de forma que não sejam necessários grandes investimentos em tecnologia para que as empresas possam usufruir dele. O presente trabalho: a) caracteriza a relevância de informatização para as MPEs e as dificuldades de acesso à tecnologia de informação; b) apresenta o desenvolvimento de sistema de gestão para micro empresas; c) propõe investigação junto à incubadora de empresas de Araraquara-SP, visando à avaliação, por parte das empresas, do sistema proposto.

\section{Palavras-chave:}

Microe Pequenas Empresas - MPEs; Sistemas deInformação; Desenvolvimento Regional; Gestão das Organizações. 
A ATIVIDADE DE EXTENSÃO NAS

INSTITUIÇÕES DE ENSINO SUPERIOR

PRIVADO: UM ESTUDO SOBRE A

COOPERAÇÃO UNIARA - MEIO PRODUTIVO

NA REGIÃO DE ARARAQUARA, SP.

Helena Carvalho De Lorenzo*

Sergio Azevedo Fonseca**

Cassiana Montesião de Sousa***

\section{Introdução}

Este texto tem o propósito de relatar e discutir os resultados de um aspecto específico de projeto idealizado e conduzido por duas instituições acadêmicas vinculadas a Universidades regionais situadas no interior do Estado de São Paulo. O projeto, desenvolvido entre os anos de 2002 a 2006, teve como objetivo principal o estudo do desenvolvimento local e as alternativas de sustentabilidade na região de Araraquara e São Carlos ${ }^{1}$. Desdobrou-se em vários subprojetos, dentre os quais o subprojeto aqui apresentado e voltado ao estudo das relações entre as Universidades regionais e o meio produtivo.

A escolha desse foco deveu-se a crescente importância da valorização das instituições de ensino e pesquisa que compõem o complexo tecido da sociedade local, que passa a demandar conhecimento em crescentes qualidade e quantidades. Assim, o estudo das Universidades enquanto instituições geradoras e difusoras de conhecimento para o meio externo e das atividades

* Docente do Mestrado em Desenvolvimento Regional e Meio Ambiente. Centro Universitário de Araraquara - UNIARA.hclorenzo@uniara.com.br

** Departamento de Administração Pública FCL/UNESP, Campus de Araraquara. saf@fclar unesp.br.

*** Bolsa de Iniciação Científica FAPESP. Curso de Administração Pública FCL/ UNESP.

1. "Políticas Públicas e Desenvolvimento Regional: identificação e avaliação de impactos econômicos e ambientais resultantes das principais transformações na estrutura produtiva da Região Araraquara - São Carlos, 1990 - 2005”. Programa de políticas Públicas FAPESP. SP. 